Utah State University

DigitalCommons@USU

$1-1-2004$

\title{
An Optical Source for Characterizing CMOS Imagers
}

Jed J. Hancock

Doran J. Baker

Gene A. Ware

Follow this and additional works at: https://digitalcommons.usu.edu/sdl_pubs

\section{Recommended Citation}

Hancock, Jed J.; Baker, Doran J.; and Ware, Gene A., "An Optical Source for Characterizing CMOS Imagers" (2004). Space Dynamics Lab Publications. Paper 54.

https://digitalcommons.usu.edu/sdl_pubs/54

This Article is brought to you for free and open access by the Space Dynamics Lab at DigitalCommons@USU. It has been accepted for inclusion in Space Dynamics Lab Publications by an authorized administrator of DigitalCommons@USU. For more information, please contact digitalcommons@usu.edu.

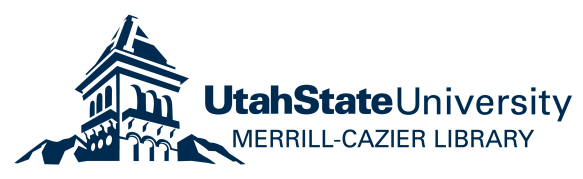




\title{
An optical source for characterizing CMOS imagers
}

\author{
Jed J. Hancock ${ }^{a}$, Doran J. Baker ${ }^{b}$, Gene A. Ware ${ }^{b}$ \\ ${ }^{a}$ Space Dynamics Laboratory, 1695 No. Research Park Way, No. Logan, UT, USA 84341; \\ ${ }^{b}$ Utah State University, Old Main Hill, Logan, UT, USA 84321-4120
}

\begin{abstract}
To characterize CMOS imagers an LED-based multi-spectral optical source system was designed and tested which is capable of illuminating a 15-mm field at a conjugate distance of $50 \mathrm{~mm}$ with 98 percent uniformity. The calibration source is comprised of an array of RGB semiconductor LEDs, an IR cutoff filter and a diffusing lens. The system is integrated into an anodized aluminum housing. The spatial uniformity of the LED optical source was compared with an optical integrating sphere and an Optoliner projection system.
\end{abstract}

Keywords: CMOS imagers, characterization, calibration, LED applications, light sources

\section{APPROACH}

To characterize the opto-electronic performance of a CMOS (complementary metal-oxide semiconductor) imager, a distributed multi-spectral light source is required to illuminate the two-dimensional array. To accomplish the characterization objective, the system needs to provide uniform illumination over the CMOS array and to give selectable red, green, and blue (RGB) spectral coverage. The design approach for the illuminator system, illustrated in Fig. 1, is to use a source array of LEDs (light emitting diodes), an infrared (IR) cutoff filter, and diffusing optics. LEDs were chosen as the source of optical radiation because of their relatively narrow bandwidth, the fast rise/fall times, compact form, efficiency and ease of intensity control.

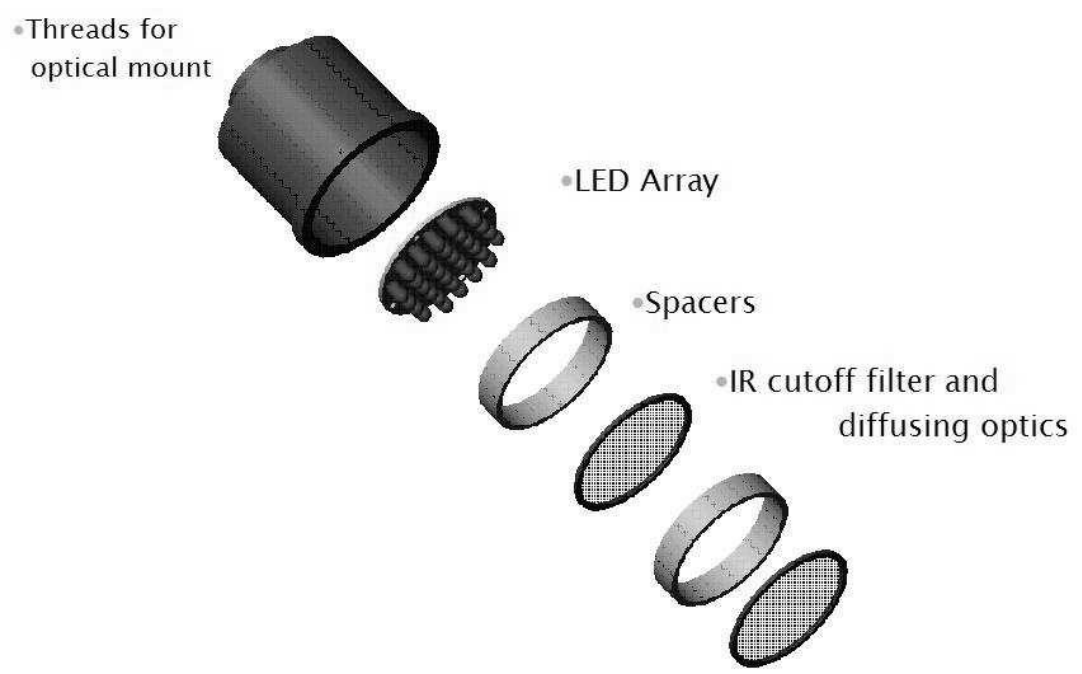

Figure 1. An exploded view of the illuminator.

The array and optics are configured into an integrated illuminator unit. The LED array is placed in the back of the light source mechanical housing. Following the array, spacers are inserted into the barrel in order to

Further author information: (Send correspondence to Jed J. Hancock)

E-mail: jed.hancock@sdl.edu, Telephone: 14357971408 
separate the LED ensemble from the IR cut-off filter. Additional spacers are placed between the IR cut-off filter and the diffusing optics. The spacers are made of nylon cut to the appropriate lengths. The illuminator system is held in place by a retaining ring fastened at the end of the barrel of the mechanical housing. The intensity of the radiated light is electronically controlled through a DC electronic driver circuit. This enables the user to set the level of the irradiance on the CMOS array under test as a function of the current through the LEDs.

\section{LED SOURCES}

The LEDs used in the light source unit are red/green/blue LEDs made by Nichia Corporation, model number NSTM515AS. The LEDs are strategically placed in a circular array to enhance the uniformity of the emitted light. The RGB LEDs have a rise/fall time faster than 1 us and enable the tester to have three selectable wavelengths of light with one LED ring. The LEDs have four leads: three separate cathodes plus a common anode. The colors red, green, and blue can be selected by simply grounding the desired cathode. The specified spectral band emission spectra of the RGB LED is shown in Fig. 2. ${ }^{1}$

\section{Spectrum}

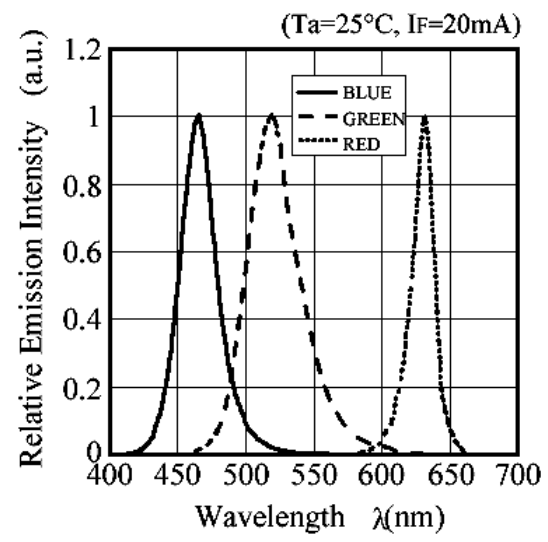

Figure 2. RGB LED emission spectrum.

LED specifications such as emission angle, intensity, emission spectrum, and lens cap must be considered when selecting LEDs for a particular application. For example, in order to produce the most spatially uniform distribution of light, one might select LEDs with an appropriate emission angle. Table 1 shows the electro-optical specifications of the RGB LEDs used in the illuminator unit. ${ }^{1}$

Table 1. RGB LED Electro-Optical Specifications

\begin{tabular}{|c|c|c|c|}
\hline & Red & Green & Blue \\
\hline Luminous Intensity (candela) & 0.18 & 0.6 & 0.13 \\
\hline Forward Voltage Drop (V) & 1.9 & 3.5 & 3.6 \\
\hline Forward Current (mA) (max) & 50 & 30 & 30 \\
\hline Emission Angle (degrees) & 80 & 80 & 80 \\
\hline Lens Cap & Diffused & Diffused & Diffused \\
\hline
\end{tabular}

The amount of light that each individual LED radiates is dependent upon the amount of current through the LED. To increase the probability of having an identical current through each LED, each LED is placed in series with a one-percent-tolerant 200-ohm resistor. The resistor also protects the LED from being damaged by an excessive amount of current. The user can set the light intensity of the source by controlling the current through 
the LED array. Different LED arrays containing LEDs with other desirable wavelengths can be substituted and inserted into the light source unit.

\section{SPATIAL CHARACTERIZATION}

In order to use the optical source to calibrate and test the performance of CMOS image sensors: (1) The pixel array must be uniformly illuminated, and (2) the spectrum of the emitted light must be known. Since the optical source is intended to uniformly illuminate an imager at a fixed distance $(\mathrm{R})$, the calibration parameter of interest is the irradiance ${ }^{2}$

$$
E=\frac{\phi}{A}
$$

as illustrated in Fig. $3 .^{3}$

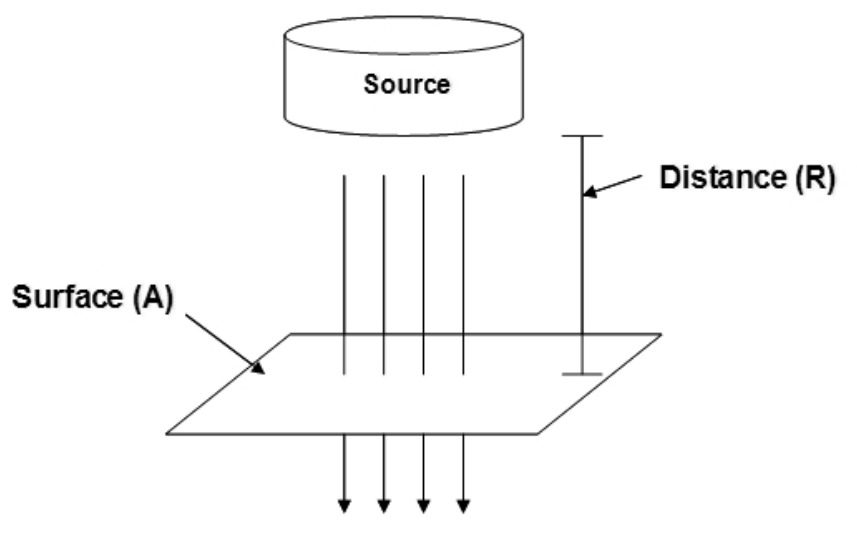

Figure 3. A surface (A) positioned a distance (R) from a radiating source of light.

The CMOS imager (or other types of detector array) to be tested is placed in the illuminated field of the optical source. The illuminated field, or scene, should be characterized in terms of spatial location for correct placement of the device under test. Characterizing the field of illumination created by the light source requires measuring the irradiance at distributed spatial locations in the illuminated scene.

In order to spatially characterize the field of illumination a calibrated photodiode is positioned normal to the projection of light from the source. Using a gimbal mount, the calibrated photodiode is moved in 1-mm increments across the illuminated scene. The photodiode is passed through the optical axis (the spatial location directly beneath the center of the radiating source). Figure 4 shows the plot of the normalized irradiance across an illuminated scene of $15-\mathrm{mm}$.

From this data it is concluded that the spatial locations of peak irradiance appropriately surround the optical axis. More importantly, it can be concluded that across an illuminated scene of $15-\mathrm{mm}$, the irradiance is within 2 percent of the maximum. In other words, if an illuminated scene with a diameter of 15-mm is centered on the optical axis; the scene will have a non-uniformity of less than 2 percent. However, the results are only valid if the illuminated scene is at a conjugate distance ranging from 50 to $75 \mathrm{~mm}$.

In order to characterize the irradiant uniformity across a larger surface area, the photodiode was successively placed: (1) Directly on the optical axis and (2) spatially displaced 15-mm away from the optical axis. Figure 5 shows the irradiance measured in 1-mm displacements away from the optical axis of the source. The plot demonstrates that a $30-\mathrm{mm}$ diameter surface centered on the optical axis will have a uniformity of approximately 95 percent.

The measurements shown in Fig. 5 were taken at right angles in directions away from the optical axis of the source. The irradiance was observed to decrease equally as a function of distance from the optical source in 


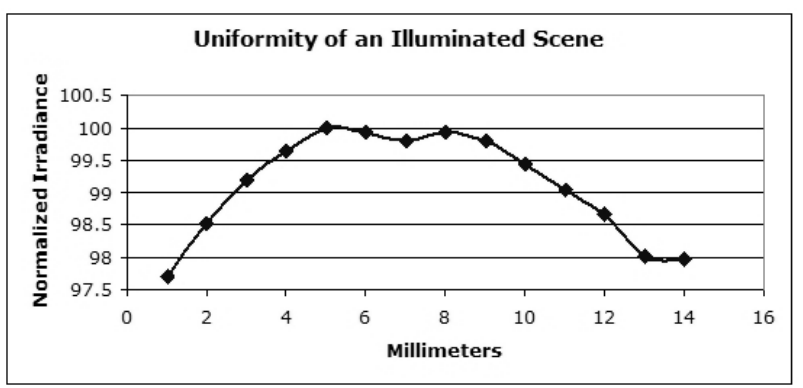

Figure 4. Normalized irradiance across a 15-mm illuminated scene centered on the optical axis of the source.

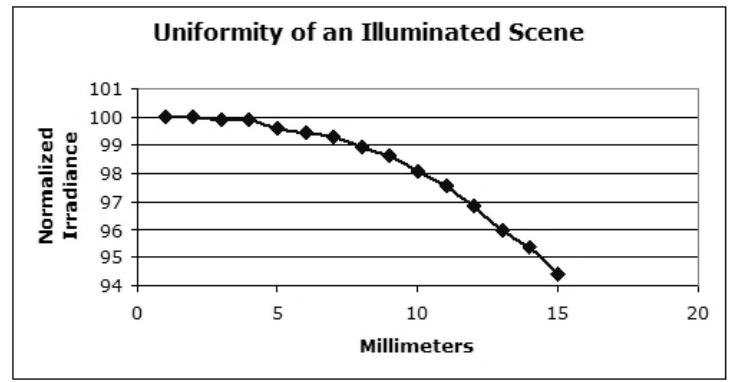

Figure 5. Normalized irradiance across a 15-mm illuminated scene away from the optical axis of the source.

directions normal to the optical axis. Therefore, it could be concluded that the irradiance pattern of the source is circular. Using these measurements, a map was made to characterize the field of illumination created by the optical source. For illustrative purposes only, Fig. 6 is an imprecise characterization map of the illuminated field of the optical source. An actual spatial illumination map can be created by an operator when placing an imager under test. The characterization map will be valid if the optical source is used at a conjugate distance between 50 and $75 \mathrm{~mm}$. From this data it is concluded that by using high angle diffusing optics and by placing the LEDs in a strategic array, the desired uniform distribution of light can be obtained. The uniformity of the optical source has been compared with commercially available light sources.

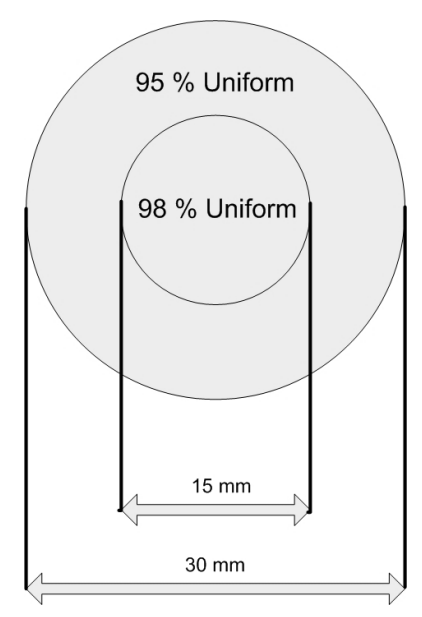

Figure 6. The optical source's characterized field of illumination. 


\section{SPECTRAL CHARACTERIZATION}

The LEDs were selected so that the optical source would be capable of emitting three distinct colors of light: red, green, and blue. To characterize the emission spectrum of the optical source, the spectral location where the peak radiant power occurred was identified for each semiconductor LED emitter. A monochromator, together with a calibrated radiometer, was used to measure the emission spectra and locate the spectral emission peaks. Commercially available optical instruments made by Oriel Instruments were used. Figure 7 illustrates the optical bench setup used to measure the spectral emission of the light source.

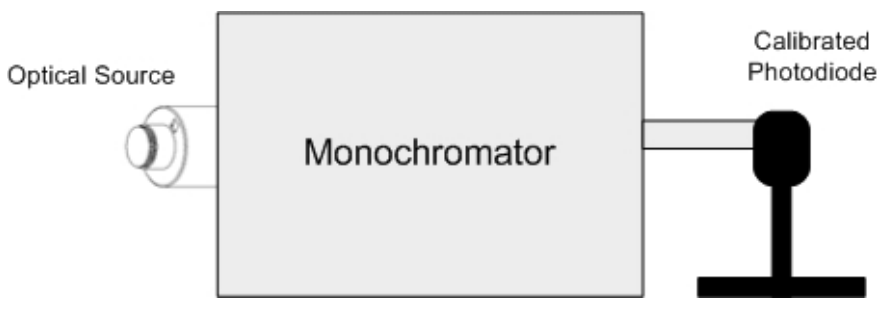

Figure 7. Instrumentation setup for characterizing the emission spectrum.

The LEDs have a narrow band emission. Each individual band in the emission spectrum of the optical source was characterized separately by directing the light from the optical source to a monochromator. After selecting a particular wavelength increment, the monochromator directs the light to a calibrated radiometer. The Oriel radiometer is a photodiode whose responsivity has been calibrated using international standards to measure the absolute spectral irradiance imposed on the photodiode versus wavelength. The wavelength where the peak emission occurs was found from recording the irradiance at each incremental band in the LED emission spectrum.

Figure 8 shows the spectral irradiance of the optical source for each color red, green, and blue, respectively. These results are summarized in Table 2 .
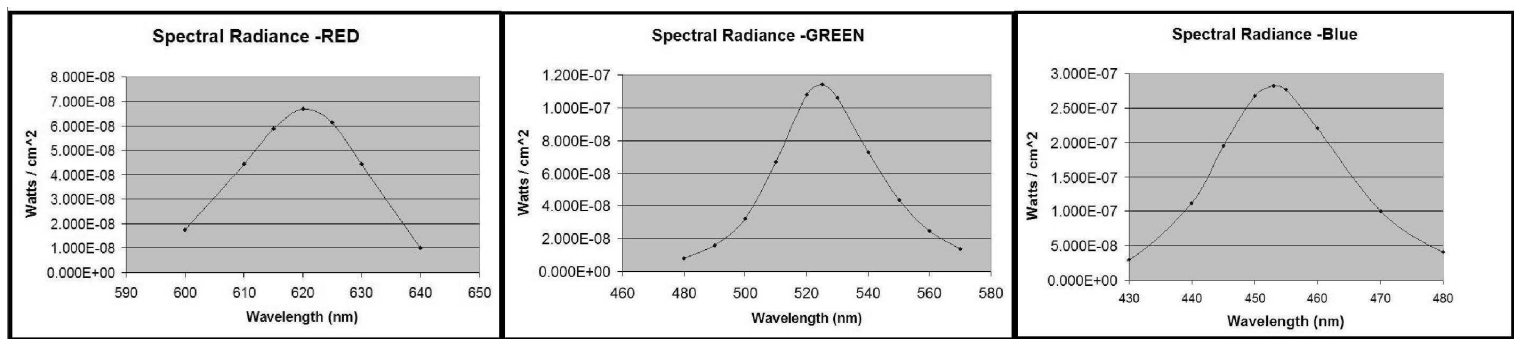

Figure 8. Red, green, and blue emission spectra of the optical source.

Table 2. Spectral Characterization of the Nichia NSTM515AS RGB LED Sources

\begin{tabular}{|c|c|c|c|}
\hline & Red & Green & Blue \\
\hline Spectral Peak (nm) & 620 & 525 & 455 \\
\hline Half Power Emission (nm) & 30 & 40 & 15 \\
\hline
\end{tabular}

\section{CALIBRATION PRODEDURE}

The optical source calibration is intended to compare the irradiant intensity of the optical source at the device under test with the input current of the LEDs in the illuminator. Calibrating the optical source in terms of current is necessary in order to adjust the current source to supply the proper amount of current to the illuminator. 
To calibrate the optical source, the irradiance was measured by integrating a CMOS image sensor at conjugate distances of 50,67.5, and $75 \mathrm{~mm}$. The sensitivity of the CMOS imager was calibrated in terms of volts per energy flux density. The flux density $\left(u J / \mathrm{cm}^{2}\right)$ upon the detector can be computed by knowing the size of the pixels in the CMOS imager array. Figure 9 shows the sensitivity of the calibrated CMOS imager as a function of wavelength.

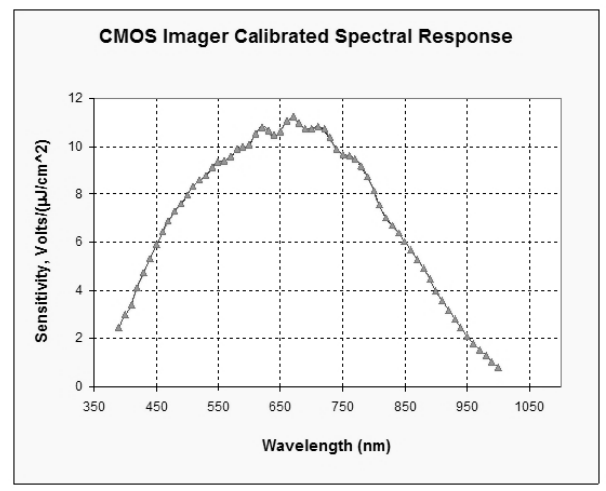

Figure 9. Responsivity of the calibrated CMOS imager.

After exposing the CMOS pixel array to the incident radiation from the light source for a specified amount of time (integration time), the image sensor output is recorded. The CMOS image sensor output voltage is then converted to irradiance by dividing the output voltage of the imager by the appropriate responsivity value. The following equation gives the conversion:

$$
E=\frac{W}{A \cdot \delta t}=\frac{V c m o s}{R}
$$

where $E$ is the irradiance in $\left(u W / \mathrm{cm}^{2}\right), W$ is the energy in $u J, A$ is the area in $\mathrm{cm}^{2}, \delta t$ is the integration time in seconds, $V c m o s$ is the CMOS image sensor output voltage, and $R$ is the calibrated responsivity $\left(V /\left(u J / \mathrm{cm}^{2}\right) \cdot \delta t\right)$, which includes the calibrated sensitivity values in Fig. 9. Using this procedure, the irradiance emitted by the LED light source from any conjugate distance can be computed in terms of input current.

The plots in Fig. 10 show the irradiance emitted by the LED optical source at three different conjugate distances as a function of LED current. Each plot gives the irradiance at the device under test for the colors red, green, and blue, respectively. The conjugate distances 50, 67.5, and $75 \mathrm{~mm}$ were chosen for the test. These dimensions correspond to the physical dimensions of the intended application. The sensitivity value from Fig. 9 was used to convert the output voltage of the calibrated CMOS imager to irradiance. Recalling from the previous section, the peak spectral radiance of the optical source occurs at the following wavelengths: $620 \mathrm{~nm}$ for red, 525 $\mathrm{nm}$ for green and $455 \mathrm{~nm}$ for blue.
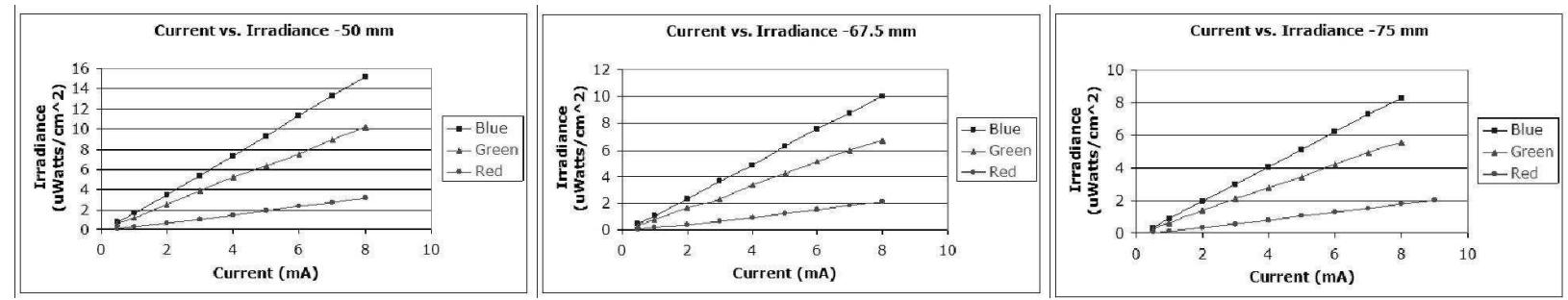

Figure 10. Current vs. Irradiance at a conjugate distance of $50 \mathrm{~mm}$.

The calibration plots indicate a linear relationship between the irradiance and the LED current. The slight non-linearity observed occurs because of the inherent electro-optical performance of the LEDs in the illuminator. 
It is also noted that any non-linear relationship between irradiance and current is compensated for in the complete optical source system because of the feedback correction achieved by using a TSL253 light-to-voltage converting sensor.

The voltage output of the pixel array is a function of the integration time, conjugate distance, and irradiance upon the array. The irradiation is controlled by the light source and the configuration. However, the integration time of the sensor is a function of the device being tested. The integration time is usually programmable and is able to be controlled within a given range depending on the specific type of CMOS imager. In order to determine the current required by the light source to completely saturate a CMOS imager, the following calibration was performed. A color CMOS image sensor was configured at a conjugate distance of $75 \mathrm{~mm}$ from the device under test, and the pixel array integration time was chosen to be $20 \mathrm{~ms}$. The color CMOS image sensor was then illuminated by the optical source using the colors red, green, and blue. The curves in Fig. 11 demonstrate the response of the red, green, and blue pixels when illuminated with their corresponding colors.

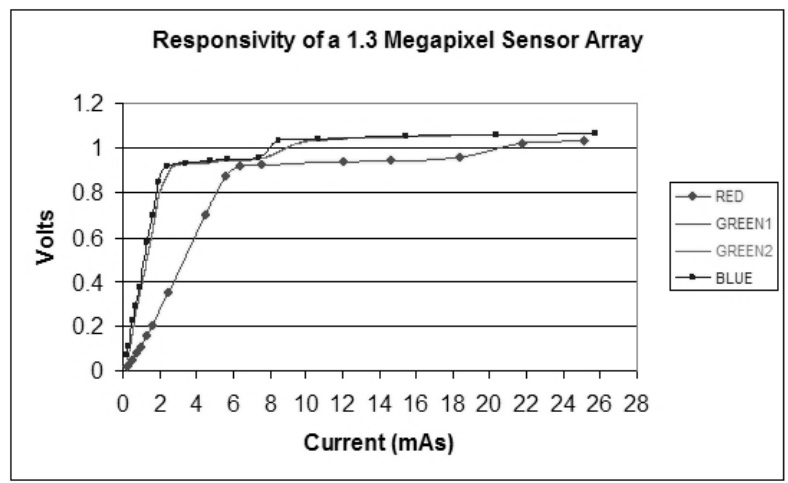

Figure 11. Current required to saturate a 1.3 megapixel CMOS imager.

It is concluded from Fig. 11 that at a conjugate distance of $75 \mathrm{~mm}$ and at an integration time of $20 \mathrm{~ms}$, the pixel array is saturated at currents much less than $20 \mathrm{~mA}$ or $4 \mathrm{uW} / \mathrm{cm}^{2}$ of red light irradiance. Because the maximum current capacity of the illuminator unit is $900 \mathrm{~mA}$, the optical source is capable of providing much more irradiance than necessary when configured at a conjugate distance of $75 \mathrm{~mm}$. This provides freedom of adjusting the conjugate distance to a much greater value and/or shortening the integration time of the pixels on the device under test. This capability is valuable for the characterization engineer because it enables the optical source to be used with custom device characterization procedures under nearly all desirable configurations.

\section{COMPARISON WITH AN INTEGRATING SPHERE}

An integrating sphere is recognized as a near ideal source of uniform illumination. It is most often used for radiometric measurements when uniformity of light is essential. An integrating sphere is a hollow sphere coated on the inside with a highly reflective white diffuse material. ${ }^{4}$ Figure 12 illustrates the operating principle of an integrating sphere. ${ }^{5}$ Light is collected in the sphere through an input port, the light is then repeatedly reflected and scattered by the inner surface of the sphere. The light exits through an output port as an almost ideal uniform beam of light. As shown in Fig. 12, a baffle is inserted inside the sphere to prevent any direct ray paths from the input port to the output port.

Comparison of the irradiant uniformity of the optical source with that of an integrating sphere is used to set a standard of performance for the optical source design. The comparison also verifies the accuracy of the characterization. The following experiment was conducted to compare the irradiant uniformity of the two sources. Each source individually illuminated a 1.3-megapixel CMOS image sensor that has an active imaging area of 6.83 by $5.45 \mathrm{~mm}{ }^{6}$ The CMOS pixel array was spatially centered on the optical axis of the source and was configured at a conjugate distance of $75 \mathrm{~mm}$. Each test was carefully configured to be identical in order to provide a valid 
comparison between the two distinct sources. While under direct illumination from an individual source, an image was captured and each individual pixel value was recorded. The pixel values from each image were then plotted in a histogram. Figure 13 contains the histogram of the CMOS imager array pixel values.

The plot labeled "Optical Sources" is a histogram of the imager pixel values when illuminated by the LED optical source. The plot labeled "Integrating Sphere" is a histogram of the imager pixel values when illuminated by the integrating sphere. Using the same CMOS imager and configuring the experiments exactly the same, an informative comparison of the histogram plots was made. Because each histogram is a distribution of all 1.3 million pixel values in the imager array, a more narrow distribution of pixel values signifies a more uniform irradiance across the array. As can be seen from the histogram in Fig. 13, the imager array pixel values have a more narrow distribution when illuminated by the optical source.

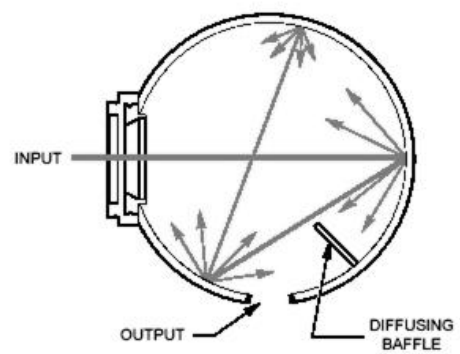

Figure 12. Principle of operation of an optical integrating sphere.

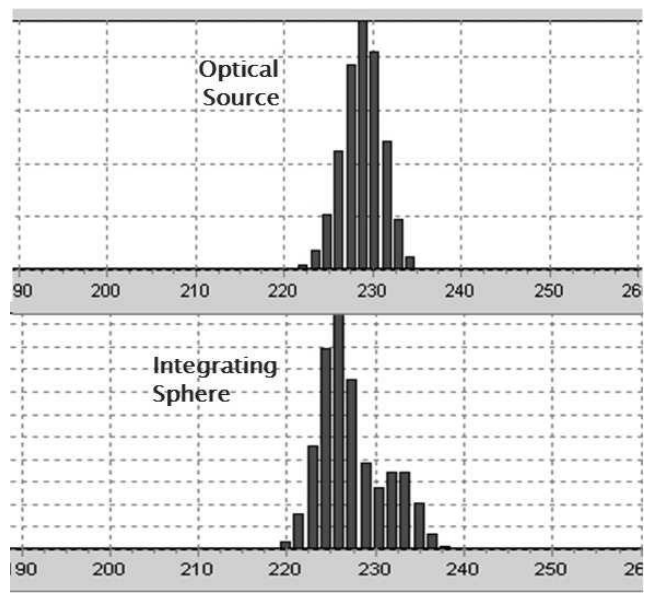

Figure 13. Histogram plots used to compare the irradiant uniformity of the LED optical source with an integrating sphere.

It is noted that the CMOS imager used in the experiment has an associated fixed pattern noise (FPN) and the spatial non-uniformity of the two distinct light sources is not identical. The imager FPN and different spatial non-uniformity of the sources are possible reasons why the LED optical source appears to illuminate the CMOS imager the most uniformly.

\section{COMPARISON WITH AN OPTOLINER PROJECTOR}

The Optoliner projection system made by Davidson Optronics is another commercially available optical source used to characterize the performance of CMOS image sensors. As shown in Fig. 14, the Optoliner projector is 
an illumination system that employs an incandescent bulb, and integrating sphere, and lens projection optics. ${ }^{7}$

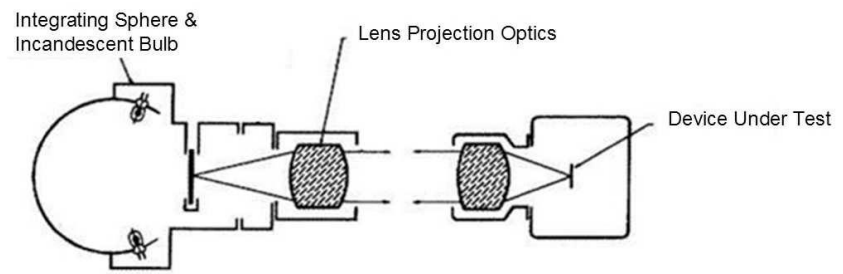

Figure 14. Davidson Optoliner projection system.

An Optoliner is used to project distortion-free images on image sensor arrays and to illuminate sensor arrays with a high degree of uniformity. The Optoliner projector serves as a good measure of comparison with the LED optical source because it is a widely used imaging instrument. In order to compare the irradiant uniformity of the Optoliner projector with the LED optical source, each experiment was configured identically. A calibrated photodiode was placed on a surface normal to the projected beam of light at a conjugate distance of $75 \mathrm{~mm}$. Using a gimbal mount, the calibrated photodiode was moved in increments of 1-mm across an illuminated scene passing through the optical axis. The irradiance detected at each 1-mm displacement was detected and is plotted in Fig. 15.

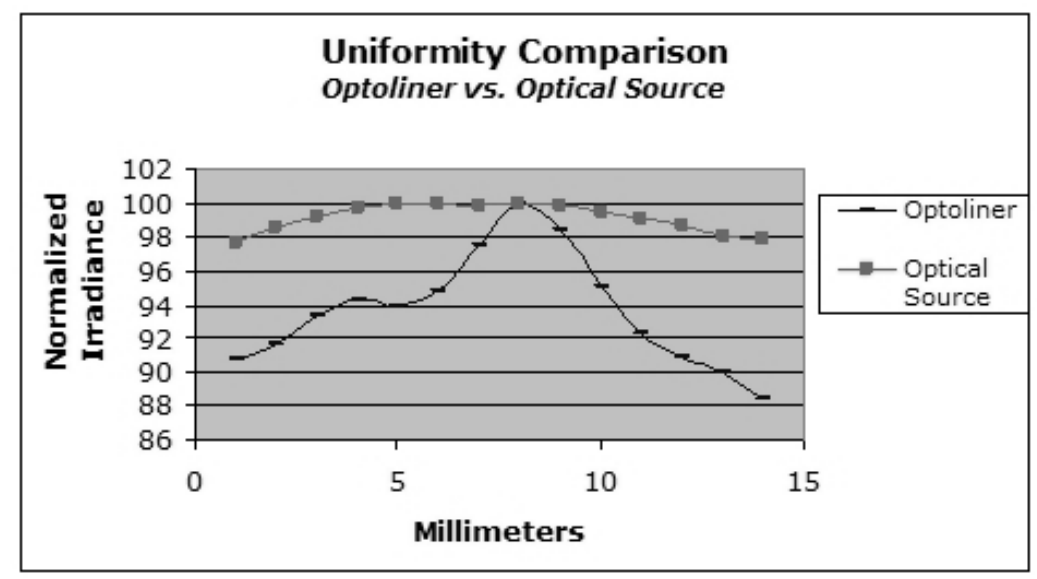

Figure 15. The irradiant uniformity of the LED optical source compared with the irradiant uniformity of the Optoliner projector.

The optical source illuminates a 15-mm diameter scene centered on the optical axis with an uniformity of 98 percent. The plot shows that the irradiance of the Optoliner projector across a 15-mm field centered on the optical axis has a uniformity of approximately 90 percent. The LED optical source has a more uniform irradiance than the Optoliner projector system.

\section{CONCLUSIONS}

The irradiant uniformity of a 15 -mm diameter illuminated scene was characterized and found to be 98 percent uniform. An illuminated scene with a $30-\mathrm{mm}$ diameter was also characterized and found to be 95 percent uniform. The irradiant uniformity of the LED optical source was compared with both an integrating sphere 
and an Optoliner projector. The experimental results indicated that the irradiant uniformity of the LED optical source is comparable with that of an integrating sphere. The irradiant uniformity of the LED optical source was found to be superior to that of an Optoliner projector. From the characterization and comparison results, it is concluded that the optical source meets the necessary specifications for characterizing CMOS imagers.

The optical source might also be characterized and calibrated under differing environmental conditions. CMOS imagers are often tested for functionality at extreme temperatures. One may consider characterizing the spectral emission and field of illumination of the LED source when it is operated at extremely high and low temperatures. The optical source may be considered as an appropriate source of irradiance when testing and characterizing other optoelectronic devices. The LED optical source could be used to characterize and/or calibrate photocells, CCDs, radiometers, etc.

\section{ACKNOWLEDGMENTS}

The support of the Rocky Mountain NASA Space Grant Consortium and Micron Technologies for working together to make the development of the optical source a success is appreciated. Special thanks is extended to the Imager Engineering group at Micron Technologies in Boise, Idaho, and Pasadena, California, for making it possible to characterize and calibrate the optical source. The help of Jennifer Blatter and Kimberly Olson is also acknowledged.

\section{REFERENCES}

1. Nichia Corporation, "Lamp Type Light Emitting Diode Product Guide." www.nichia.com, 2003.

2. A. Ryer, Light Measurement Handbook, pp. 9-32. Newburyport, MA, International Light, 1997.

3. D. C. O'Shea, Elements of Modern Optical Design, pp. 87-107. New York: John Wiley and Sons, 1985.

4. W. J. Smith, Modern Optical Engineering, pp. 247-248. New York: McGraw Hill, 2000.

5. Oriel Instruments, "Integrating Spheres." www.oriel.com, 2003.

6. Micron Technologies, "Product Specifications." http://www.micron.com, 2003.

7. Davidson Optronics Incorporated, "Optoliner Projector." www.davidsonoptroni-cs.com, 2003. 